\title{
EVOLUCIÓN HISTÓRICA DEL MÉTODO ENFERMERO
}

\author{
Amparo Nogales Espert
}

Prof. Titular Historia de la Enfermería. Facultad de Ciencias de la Salud Universidad Rey Juan Carlos. Alcorcón, Madrid

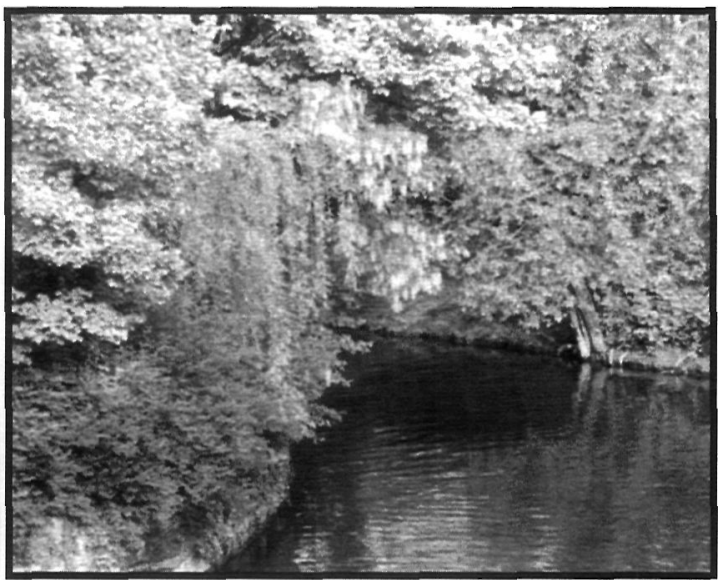

EVOLUTION OF THE NURSING METHOD

$\mathrm{T}$ The present article tries to give a brief look down on the historic evolution of the nursing method to simply give evidence of the existence of a formal and continuous - never interrupted - education in nursing since long time ago, according to the different historic moments that nursing education suffered from higher or lower influence in society depending on the personalities implied at each time.

As primary sources we have handled the Nursing Regulations of the Hospital Provincial de Valencia in 1897 in the custody of the Library Archives in the Diputación Provincial de Valencia.

As a fundamental conclusion, we underline the paramount need to maintain a real service attitude to patients and society which are always basic to define and justify the existence of our profession.

Key words: historic, nursing evolution, influence, conclusion

\section{RESUMEN}

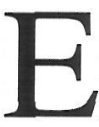

$n$ la presente intervención pretendemos realizar un breve repaso de la evolución histórica del método enfermero, para poner de manifiesto la existencia de una enseñanza reglada de la enfermería desde los más remotos tiempos, con una continuidad que se ha prolongado de manera más o menos notable según distintos momentos históricos, y también bajo la influencia de determinadas personalidades relevantes de la enfermería.

Como fuentes primarias hemos utilizado el reglamento de las enfermerías del Hospital Provincial de Valencia de 1897, custodiado en el Archivo de la Diputación Provincial de Valencia.

Destacamos como conclusión fundamental la necesidad de mantener entre nuestra comunidad de estudiantes y profesionales de enfermería una actitud de servicio hacia los pacientes y hacia la sociedad, por constituir un poderoso elemento justificador y definitorio de nuestra profesión.

El título de esta Mesa Redonda, "Evolución histórica del Método Enfermero", nos lleva a unas reflexiones de gran calado, como acabamos de oír en la exposición de la Profesora Hernández Martín.

También nos lleva esta propuesta sobre la evolución del método enfermero, a plantearnos varios interrogantes sobre cómo ha ido transformándose la enseñanza de la enfermería en el devenir de la historia; preguntas a las que intentaremos encontrar alguna respuesta en esta intervención.

Método, del griego "métodos" es el modo de decir o hacer con orden, el procedimiento que se sigue en las ciencias para hallar la verdad y enseñarla, en definitiva, el camino que en las distintas disciplinas se recorre durante el proceso de su enseñanza-aprendizaje.

Trataremos de aproximarnos a algunos momentos de la historia de la enfermería que por su representación humana o institucional, constituyeron destacados puntos de apoyo, a patir de los cuales podría decirse que la enfermería salió fortalecida en sus conocimientos y en la enseñanza de los mismos. Son los siguientes:

1.- Las Matronas Romanas. Su formación y su papel como docentes. 
2.- Fundación del Hospital Basilias en el año 370. Influencia por sus dimensiones y características en la formación de las enfermeras.

3.- Órdenes y Congregaciones dedicadas a la enfermería:

- Hermanos de San Juan de Dios

- Mínima Congregación de Hermanis

Enfermeros pobres, u "Obregones"

- Hijas de la Caridad.

4.- Instituto de Diakonisas para la formación de enfermeras.

5.- Florence Nihtingale, impulsora de la enseñanza moderna de la enfermería.

6.- Reglamento de las Enfermerías del Hospital Provincial de valencia, 1897. Formación de Practicantes.

7.- Libros de texto para la enseñanza de la enfermería.

8.- Normativas y Reglamentos docentes para el ejercicio de la profesión.

1.- Las Matronas Romanas. Su formación y su papel como docentes: En el siglo IV de nuestra era tres grandes mujeres de la aristocracia romana, por inspiración cristiana, pusieron al servicio de la enfermería su cultura y preparación intelectual, y también su enorme fortuna. Han recibido el nombre genérico de Matronas Romanas, destacando especialmente Marcela, Fabiola y Paula.

Marcela, a quien se atribuye una notable inteligencia, desarrolló una gran labor docente, e instruyó a sus seguidoras en el cuidado de los enfermos.

Fabiola: Recibió formación sanitaria de las fuentes de Sorano de Efeso y de Galeno.

Enseñó reglas sobre el cuidado de los recién nacidos, ancianos y enfermos dependientes (minusválidos),

Sistematizó y enseñó reglas sobre alimentación, higiene y Salud Pública, potabilización de aguas residuales y cementerios subterráneos.

En el año 390 fundó con los bienes de su fortuna el primer hospital gratuito de Roma. En él trabajó personalmente como enfermera y organizó los cuidados de forma que los pacientes eran transportados al hospital y allí recibían asistencia y alimentación adecuada, consejos y atenciones a sus necesidades específicas.

Paula: Perteneció a una de las familias romanas más antiguas y nobles. Dotada de una gran capacidad intelectual y con una vasta cultura, estudió varias lenguas, hebreo y griego y sus grandes conocimientos la convirtieron en una de las mujeres más cultas de este período de la historia.

Atraída por la enfermería en su objetivo de ayuda a los más necesitados, estudió con Marcela de quien aprendió de forma sistemática, sobre el cuidado de los enfermos.

Posteriormente se convirtió en maestra, instruyendo a las enfermeras de una forma ordenada.

Como carácter definitorio de la docencia, enseñó la enfermería como un arte diferenciado, dejando bien clara su diferenciación como oficio en ciernes, con independencia de su objetivo de servicio general a los pobres.

Encontramos descrita en esta etapa una enseñanza planificada de la enfermería, con docencia extendida por el mundo romano, años 300 a 400 d.C.

2.- Fundación del Hospital Basilias: En la historia de la enfermería existe la certeza del papel insustituible de las enfermeras desarrollado en los hospitales, así como la transformación indiscutible que la enfermería experimentó, a través de las actividades asistenciales desarrolladas en estos centros asistenciales. Pues bien, uno de los primeros y más famosos hospitales que se erigió fue el llamado Basilias, fundado por San Basilio el Grande en Cesarea el año 370.

Se trataba de una institución de enormes proporciones, a modo de una ciudad autosuficiente que, en edificios se parados dentro de un gran recinto, atendía todas las necesidades sanitarias: prevención, asistencia, tratamiento y servicio social.

Contaba con un personal numeroso y tipificado: médicos, y enfermeros, residentes en dependencias del recinto hospitalario y personal encargado de otras actividades, como el transporte de enfermos.

Incluso hoy nos parece sorprendente la variedad de dependencias e instalaciones desde las que se daba asistencia a diferentes necesidades específicas de cuidados:

Orfanato y asilo para lactantes y niños

Departamento de dementes 
Hospital para enfermos y minusválidos

Edificio para ancianos

Escuela de artesanía y oficios para impedidos físicos.

Todas las personas residentes aptas para trabajar eran empleadas en sus dependencias de restauración: cocina, lavandería, granja, talleres (calzado, ropa), herrería (fundición). Probablemente estamos hablando del primer proyecto de Terapia Ocupacional llevado a la práctica en la historia.

No hemos encontrado datos sobre la existencia de enseñanza organizada de enfermería en este hospital de inmensas proporciones. Sin embargo nos preguntamos: ¿Hubiera funcionado esta institución sin una minuciosa organización donde las funciones de enfermería se aprendieran y desempeñaran con exactitud?. ¿Habría existido una asistencia correcta en las distintas especialidades y departamentos de no contar con un personal de enfermería adecuadamente preparado y al frente de unas responsabilidades bien delimitadas y repartidas? ¿ Hubiera podido funcionar este gran hospital sin una coordinación escrupulosa de todos los oficios y una exigencia indiscutible del correcto cumplimiento de las obligaciones de todos los miembros de su personal?

Podemos hablar, por tanto, de la muy probable existencia de una metodología en la organización y en el cumplimiento de las funciones de enfermería, en este gran hospital del año 312 .

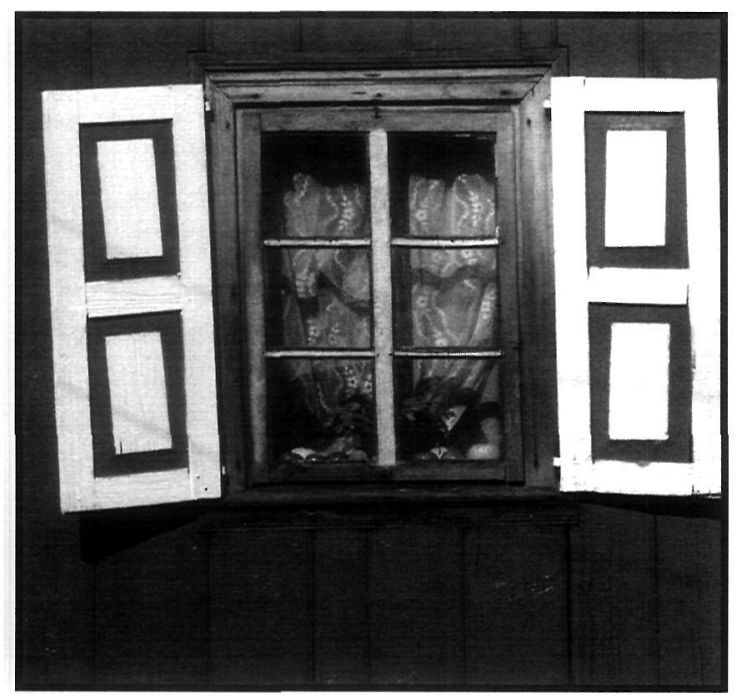

\section{3.- Órdenes y Congregaciones dedicadas a} la enfermería:

3.1. San Juan de Dios: tras la recuperación de su enfermedad, vivió en el hospital que le dio asistencia, y allí aprendió los cuidados de enfermería. A su muerte, en 1550, había fundado un hospital y contaba con un grupo de compañeros dispuestos a asumir su ideal de vida y ayuda a los enfermos.

De 1550 a 1572 en una segunda etapa de expansión de su obra, fueron creándose diversos centros asistenciales no solo en Andalucía, sino también en Madrid y Toledo.

La expansión internacional de esta obra creadora sucede entre 1572 y 1596; nacerán gran número de fundaciones en la península, y en Italia crearan hospitales partiendo del primero levantado en Nápoles, seguidamente en Roma y con posterioridad once hospitales más en otras tantas ciudades italianas.

A partir de 1596 la Orden de San Juan de Dios creó casas-hospitales prácticamente en todas las ciudades más importantes del Nuevo Mundo. Al mismo tiempo se extienden los hospitales de la Orden por las ciudades más destacadas de Europa.

La consideración de estos datos nos permite imaginar la existencia de un esquema de planificación y organización del funcionamiento de estos hospitales, en los que debía de reproducirse con todo rigor, el mismo proyecto que había asegurado el éxito en todos los hospitales anteriores y que debía resumirse de la siguiente forma:

Buena planificación y organización del trabajo. Una correcta administración.

Conocimiento y buen ejercicio de la práctica de los cuidados.

Puede sobreentenderse sin duda la necesaria existencia de una programada enseñanza, con la que debía prepararse a los hermanos de la Orden para convertirse en enfermeros capaces de cuidar eficazmente a los enfermos en las salas de sus hospitales.

3.2. La Mínima Congregación de Hermanos Enfermeros Pobres, también conocida como Obregones, fue fundada por Bernardino de Obregon, el cual, antes de convertirse en fundador de un hospital, pasó un tiempo, desde 1567 a 1579 , dedicado doce años al aprendizaje del cuidado de los enfermos en distintos hospitales. 
Los siete años siguientes, desde 1579 a 1587 , los dedicó a la fundación de un hospital, y a ocuparse en la dirección de otros varios hospitales, de esta forma estuvo encargado del funcionamiento del Hospital General de Madrid.

Sus largos años de experiencia en los hospitales le permitieron conocer la forma en que se prestaban los cuidados y también sus dificultades. Fundó una Congregación de Hermanos a los que tuvo que formar en la enfermería, hasta adquirir la experiencia necesaria para actuar directamente en los cuidados a los enfermos.

3.3. Hijas de la Caridad: San Vicente de Paul (1576 - 1660). Creó una Sociedad de Damas que llamó "Cofradía de la Caridad", y también "Damas de la Caridad", para atender a los enfermos y cuidarlos en sus casas. Madame Le Gras, más tarde Santa Luisa de Marillac se encargó de instruir a las mujeres que se sumaban al nuevo proyecto de San Vicente de Paul de fundar en 1633 una Orden de Enfermería que, tras las denominaciones anteriores fue llamada "Hermanas de la Caridad". Las jóvenes unidas a este proyecto debían reunir unas cualidades: inteligencia, educación e interés sincero por los enfermos.

En la formación de las Hermanas o "Hijas de la caridad", se estableció un sistema con una sola directriz, o línea muy clara de enseñanza y una organización:

Dos meses de prueba para las aspirantes

Un periodo de preparación de 5 años

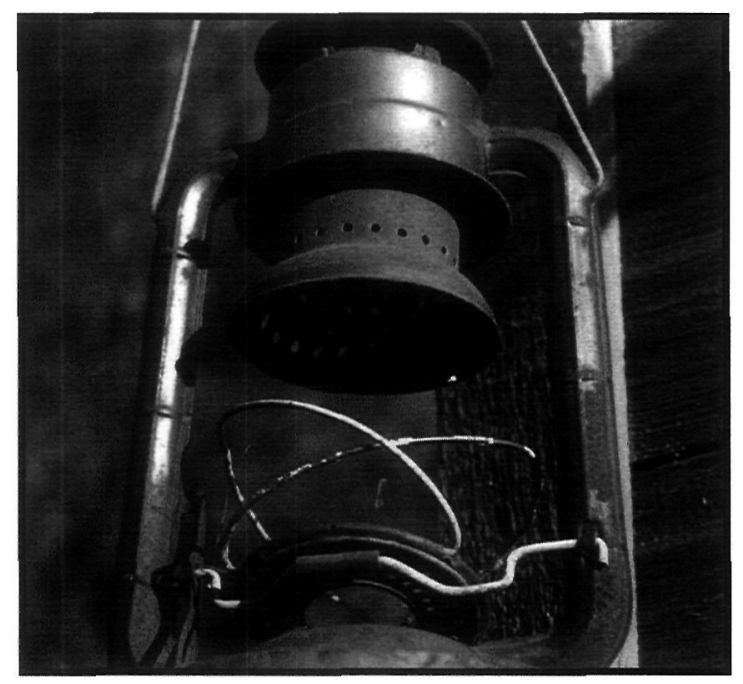

Reanudación de votos anualmente y libertad para abandonar la institución cuando lo desearan

Uso de un uniforme: túnica azul o gris, delantal, cuello blanco y tocado blanco o cornete.

Se trataba ya de una formación organizada y planificada en contenidos y tiempo de preparación.

\section{4.- Instituto de Diakonisas para la formación} de enfermeras: En 1836 el pastor protestante Theodor Fliedner fundó un Instituto de Diakonisas con un pequeño hospital para atender a enfermos. se trataba en realidad de una Escuela de formación de Enfermeras Diakonisas.

Esta escuela planificó una enseñanza reglada con un programa de 3 años de duración en el que se enseñaba la práctica de la enfermería mediante una rotación por los servicios hospitalarios y las distintas salas de hombres, mujeres, niños, enfermedades infecciosas, convalecientes y salas de diakonisas enfermas. También se practicaba la asistencia a los enfermos en sus domicilios, o enfermería domiciliaria.

Así mismo se realizaba un aprendizaje teórico. Entre los temas del programa se enseñaba farmacia, conocimientos de ética y doctrina religiosa. En los textos de formación se especificaba el principio de que las enfermeras debían cumplir exactamente las ordenes del médico, único responsable en último término, de la asistencia a los enfermos.

El trabajo se llevaba a cabo en los distritos de la ciudad (salud pública), o atención pública domiciliaria y en los hospitales.

Las enfermeras diakonisas llevaban como atuendo un uniforme distintivo: bata de algodón azul, delantal blanco, cuello vuelto, cofia blanca (volante en torno al rostro), largo manto negro y toca sobre la cofia.

\section{5.- F. Nightingale, impulsora de la enseñan-} za moderna de la enfermería: Florence Nightingale tuvo una larga experiencia formativa. Primero estudió con las Diakonisas en Alemania y con las Hijas de la Caridad en París. Adquirió así mismo una experiencia práctica muy importante, al mando de un grupo de enfermeras en el Hospital de Scutari en Turquía, transformando totalmente con sus conocimientos los cuidados de enfermería a los soldados heridos. 
En 1860 fundó en Londres una Escuela de Enfermería que llevaría su nombre, la "Escuela Nihtingale", con el objeto de formar enfermeras para trabajar en hospitales, en distritos o salud pública y como docentes.

La duración reglada de los estudios comprendía tres años, uno teórico y dos años de prácticas de aprendizaje en hospital.

La Escuela Nightingale imprimió un nuevo estilo para la enfermería y para las enfermeras. De sus aulas salían formadas verdaderas líderes muy solicitadas por instituciones de diferentes países.

\section{6.- Reglamento de las Enfermerías del} Hospital Provincial de Valencia, 1897. Formación de Practicantes: Respecto a referencias sobre metodología de la enseñanza de la enfermería en relación con los trabajos de investigación que en estos momentos realizamos podemos decir que, en el Reglamento de las Enfermerías del Hospital Provincial de Valencia de 1897, en el capitulo 8 que trata de los Practicantes Supernumerarios, concretamente en el Artículo 74 se habla del examen previo que deberían superar estos practicantes para obtener una plaza en el hospital. El tribunal de este examen estaría compuesto por los profesores de Beneficencia indicados por la Dirección del Hospital. Tras superar el examen se enviaba la propuesta de admisión a la Diputación, donde se procedía a otorgar el nombramiento definitivo.

En estos momentos además de los practicantes, trabajaban en el Hospital las Hijas de la Caridad, desempeñando la mayor responsabilidad en el cuidado de los enfermos de la sala. Su cargo recibía el nombre de "cabeza de sala", contando con "ayudantes" o subalternos para la atención generalizada a los hospitalizados, con los propios enfermeros, y con otras Hijas de la Caridad trabajando también como enfermeras.

Los enfermeros eran considerados "verdaderos sirvientes" de las salas y enfermos, y trabajaban bajo la vigilancia, inspección y dirección de las Hijas de la Caridad.

Hay que tener en cuenta, en relación con el tema que nos ocupa, el carácter docente del Hospital Provincial, en el que Profesores y Catedráticos de la Facultad de Medicina impartían docencia a los estudiantes en las salas de enfermos del hospital, su lugar de prácticas habitual, e incluso el recinto hospitalario contaba con aulas donde se daban clases teóricas a estos estudiantes. No es descabellado pensar, teniendo en cuenta además la formación reglada a que estaban acostumbradas las Hijas de la Caridad, que alguna formación recibieran así mismo las enfermeras, aunque no conste textualmente.

Hemos de considerar por otra parte el importante número constituido por el personal asistencial. En 1873 las personas encargadas de la asistencia y cuidados a los enfermos en el Hospital Provincial de Valencia eran las siguientes: 1a Superiora e Hijas de la Caridad, en número de 45; practicantes, 11; enfermeros, 10; enfermeras 4; ayudantes de enfermos dementes, 15; aparatistas, 1 ; barberos, 2 . Sin duda se requería, además de una buena organización, la necesidad de una adecuada formación previa, que no debió de faltar para asegurar la práctica de unos cuidados efectivos.

7.- Libros de texto para la enseñanza de la enfermería: La producción literaria de obras de enfermería en número creciente según avanza el tiempo nos indica la tendencia a dirigir la enseñanza acompañándola no solamente de un aprendizaje práctico, sino también de conocimientos teóricos. Los libros más destacables desde el siglo XVII hasta principios del XX son los siguientes:

Instrucción de enfermeros. Para los Hermanos Obregones, de la Congregación de Bernardino de Obregón, 1621.

Directorio de enfermeros y artífice de obras de caridad para curar las enfermedades del cuerpo. Simón López, siglo XVII.

Manual para el servicio de los enfermeros. M. Carrere, 1786.

El arte de la enfermería. José Bueno González. Hermano de San Juan de Dios, 1833.

Manual de la carrera de enfermeras para el uso de las Hijas de la Caridad, 1917.

8.- Normativas y Reglamentos docentes para el ejercicio de la profesión: En cuanto a la reglamentación de los estudios de las profesiones sanitarias, durante el siglo XVIII el Tribunal del Protomedicato continuó regulando el ejercicio pro- 
fesional mediante la concesión de cartas de autorización.

En el siglo XIX las Facultades de Medicina y los Reales Colegios de Cirugía tendrán bajo su responsabilidad el otorgamiento de autorizaciones para ejercer.

En las mismas Ordenanzas y Constituciones de los hospitales podemos encontrar la existencia de una preocupación por la formación de los enfermeros, como en las Constituciones del Hospital Real de Santiago de Compostela, 1590, o las Ordenaciones del Hospital de Nuestra Señora de Gracia en Zaragoza, 1755 y 1767.

A partir de la segunda mitad del siglo XIX se tenderá hacia la institucionalización de la enseñanza, ya de una forma sistematizada, comenzando por los practicantes y matronas.

\section{CONCLUSIÓN}

Como conclusión resaltamos los siguientes aspectos:

Existencia de una enseñanza reglada desde la antigüedad, para transmitir los conocimientos de la enfermería.

Exigencia de ciertas cualidades para ejercer la enfermería, basadas en la existencia en las aspirantes de un interés por ayudar al enfermo.

Elaboración de libros de texto para la formación de enfermeros.

Finalmente podemos decir, respecto a la metodología docente normalmente empleada en la actualidad, que existe en nuestra comunidad de estudiantes y profesionales la necesidad de mantener una actitud de servicio hacia los pacientes y hacia la sociedad. Y que tal vez desde la docencia no hemos encontrado todavía la forma de transmitirla con la nitidez adecuada en razón de su importancia, puesto que ello implica el necesario desarrollo de cualidades y valores que resultan irrenunciables en nuestra profesión, porque constituyen un poderoso elemento justificador y definitorio de la enfermería.

\section{BIBLIOGRAFÍA}

- Archivo Diputación Provincial de Valencia (1897). Reglamento de las enfermerías del Hospital Provincial, 1897. Signatura I - 5.1/C-2 Legajo 34. Fuentes primarias.
- Donahue, P. (1985) Historia de la Enfermería. Doyma, Barcelona: 107-115.

- Fernández Doctor, A (1987). El Hospital Real y General de Nuestra Señora de Gracia de Zaragoza en el siglo XVIII. Institución Fernando el Católico. Zaragoza: 325-335.

- García Martínez, A. et al (1996) "Un Siglo de Oro para la enfermería española (1550-1650):I. Aparición de ordenes y manuales de enfermería". En: HERNANDEZ MARTIN, F. Historia de la enfermería en España. Síntesis. Madrid: 137-145.

- García Martínez, A. et al. (2001) Directorio de enfermeros y artífice de obras de caridad para curar las enfermedades del cuerpo. Simón López, siglo XVII. Network Salud S.A. Madrid.

- Muñoz Miguel, E. (1751) Recopilación de las Leyes, Pragmáticas Reales, Decretos y Acuerdos del Real Proto-Medicato. Valencia. Servicio reproducciones Librerías París-Valencia, 1991: 109-111; 308-316.

- Nogales Espert, A. (1996) "Los hospitales medievales y la enseñanza medieval de la medicina". En: HERNANDEZ MARTIN, F. Historia de la enfermería en España. Síntesis. Madrid: 95-110. 